\title{
Emotional stimuli modulate readiness for action: A transcranial magnetic stimulation study
}

\author{
Anouk M. van Loon, Wery P. M. van den Wildenberg, And Anda H. van Stegeren \\ University of Amsterdam, Amsterdam, The Netherlands \\ GREg HAJCAK \\ Stony Brook University, Stony Brook, New York
}

AND

K. RICHARD RIDDERINKHOF

University of Amsterdam, Amsterdam, The Netherlands

\begin{abstract}
Emotional stimuli may prime the motor system and facilitate action readiness. Direct evidence for this effect has been shown by recent studies using transcranial magnetic stimulation (TMS). When administered over the primary motor cortex involved in responding, TMS pulses elicit motor-evoked potentials (MEPs) in the represented muscles. The amplitudes of these MEPs reflect the state of corticospinal excitability. Here, we investigated the dynamic effects of induced emotions on action readiness, as reflected by corticospinal excitability. Subjects performed a choice task while viewing task-irrelevant emotional and neutral pictures. The pattern of MEP amplitudes showed a typical increase as the TMS pulse was presented closer in time to the imminent response. This dynamic pattern was amplified by both pleasant and unpleasant emotional stimuli, but more so when unpleasant pictures were viewed. These patterns present novel evidence in support of the notion that emotional stimuli modulate action readiness.
\end{abstract}

The ability to quickly identify emotionally salient information in the environment and to form rapid and appropriate behavioral responses is critical to survival (Darwin, 1872/1965). The attribution of value to external events refers to an organism's ability to sense whether events in its environment are more or less desirable. Within this framework, emotions represent complex psychological and physiological states that are generated as a reaction to objects or events that are relevant to the individual's concerns (Frijda, 1986). The cognitive appraisal of such objects or events exerts a (selectively) potentiating effect: The detection of motivationally relevant stimuli in the environment mobilizes the body for action (Frijda, 1986, 2007). In this sense, emotions can be viewed as concern-related dynamic changes in action readiness (see also Lewis, 2005).

Behavioral findings reported by Duckworth, Bargh, Garcia, and Chaiken (2002) showed that emotions influence the action system in a specific way. They used an approach/ avoidance paradigm and demonstrated that, in the approach condition, responses were faster to positive novel stimuli than to negative novel stimuli. In the avoidance condition, this pattern was reversed. Furthermore, Coombes, Gamble, Cauraugh, and Janelle (2008) found an effect of emotional stimuli on force control. Both unpleasant and pleasant stimuli led to a greater force production and attenuated force decay, as compared with neutral stimuli. More direct evidence that emotional stimuli prime or facilitate action has been provided by studies using transcranial magnetic stimulation (TMS) - a noninvasive tool that discharges a magnetic pulse at the scalp surface (Avenanti, Bueti, Galati, \& Aglioti, 2005; Avenanti, Minio-Paluello, Bufalari, \& Aglioti, 2009; Baumgartner, Willi, \& Jäncke, 2007; Fecteau, Pascual-Leone, \& Théoret, 2008; Hajcak et al., 2007; Oliveri et al., 2003; Schutter, Hofman, \& Van Honk, 2008). Underneath the scalp, the magnetic pulse induces electric fields that cause neurons to depolarize (Bohning, 2000). If TMS is applied to the motor cortex (M1), it is possible to quantify corticospinal excitability by the magnitude of the motor-evoked potential (MEP; Terao \& Ugawa, 2002), which can be derived from the electromyogram (EMG) measured over the muscles that are represented. This specific form of TMS is referred to as motor-TMS.

Oliveri et al. (2003) stimulated both the primary motor cortex (M1) and the supplementary motor area with TMS. They showed MEPs of smaller amplitude for actively viewed neutral stimuli than for negative emotional stimuli, but only when the supplementary motor area was stimulated together with M1, not when the areas were stimulated separately. Hajcak et al. (2007) stimulated only M1 and observed that MEP amplitude was modulated by the valence of emotional stimuli. Hajcak and colleagues found enhanced MEP amplitudes when subjects were 
passively viewing emotional pictures, as compared with neutral ones. They concluded that emotional processing has direct effects on a centrally mediated measure of corticospinal excitability.

A recent TMS study demonstrated that corticospinal excitability was selectively enhanced by fearful facial expressions, as compared with happy and neutral expressions (Schutter et al., 2008). In addition, Baumgartner et al. (2007) simultaneously presented musical and pictorial stimuli of congruent emotional valence to induce an emotional state in their TMS study. They observed that this combined condition enhanced MEP amplitudes, whereas this enhancement was not seen when the visual and auditory stimuli were presented separately. This relates to a recent observation that a worry induction elicited larger corticospinal motor responses than did mental arithmetic (Oathes, Bruce, \& Nitschke, 2008). Other TMS studies demonstrated an effect of pain perception on corticospinal excitability. Observation of a painful stimulus reduced the amplitude of the TMS-induced MEP. These results were also related to personality traits, individual differences, and subjective states (Avenanti et al., 2005; Avenanti et al., 2009; Fecteau et al., 2008). In the study by Fecteau and colleagues, subjects scoring high on a coldheartedness measure displayed the greatest reduction of cortical spinal excitability, as compared with subjects with a low score on this scale. Avenanti et al. (2009) found that the somatomotor response to painful stimuli was lower in subjects with high trait- and state-personal distress and higher if subjects scored high on trait-cognitive empathy.

In sum, initial evidence has been reported in support of the claim that emotional stimuli or emotional state inductions modulate readiness of the action system, measured in terms of corticospinal excitability. This may reflect a short-lived emotion-induced effect on cortical arousal (on the order of seconds; Hajcak et al., 2007) or a more dynamic phasic effect, specifically aligned in time with the time of responding (Frijda, 2007; Lewis, 2005). Whereas previous designs did not allow specification of phasic effects (Avenanti et al., 2005; Avenanti et al., 2009; Baumgartner et al., 2007; Fecteau et al., 2008; Hajcak et al., 2007; Oathes et al., 2008; Oliveri et al., 2003; Schutter et al., 2008), we examined the temporal dynamics of the effect of emotion on action readiness.

\section{Dynamics of Action Readiness}

The dynamics of action readiness, as reflected by MEP amplitude, have been revealed in previous motor-TMS studies with nonemotional stimuli. Using single-pulse TMS applied over M1 at various time intervals between the presentation of the stimulus and the execution of the response in a simple reaction time (RT) protocol, several motorTMS studies described a gradual increase in corticospinal excitability, starting 80-120 msec prior to movement onset and rising to a maximum just prior to the time of overt responding (e.g., Leocani, Cohen, Wassermann, Ikoma, \& Hallett, 2000; Nikolova, Pondev, Christova, Wolf, \& Kossev, 2006; Rossini, Zarola, Stalberg, \& Caramia, 1988). Building on this paradigmatic approach, our present aim was to examine how this dynamic buildup of corticospinal excitability is modulated by emotional valence. More specifically, we expected that positive and negative emotional stimuli would be associated with higher MEP amplitudes (reflecting increased action readiness) and with a steeper rise of MEP amplitudes (reflecting the dynamics of action preparation) than would neutral stimuli.

Furthermore, we tested the effect of emotional valence on corticospinal excitability when task-irrelevant emotional stimuli were used. The studies mentioned above presented emotional stimuli that were task relevant. A behavioral study performed by Erthal et al. (2005) suggested that task-irrelevant emotional stimuli can also influence the response system. Their subjects performed a task in which a central picture and two peripheral bars were presented. The instruction was to ignore the task-irrelevant central picture and to respond as quickly and accurately as possible to the peripheral bars, indicating whether the bar orientations were similar or not. The central pictures had either a neutral emotional valence or an unpleasant emotional valence. RTs to the bar orientation were longer when the subjects were presented with the unpleasant pictures than when the central picture was of neutral valence.

To investigate the temporal dynamics of the effect of emotional task-irrelevant stimuli on action readiness, we conducted a new experiment that combined the methods used by Erthal et al. (2005) and Hajcak et al. (2007). We used a choice RT task, in which subjects issued a left- or right-hand response on every trial. The emotional stimuli that were presented were derived from the International Affective Picture System (IAPS). The pictures were task irrelevant, similar to the emotional stimuli in the study by Erthal et al. Presenting TMS over the hand area of the left M1 allowed us to examine the excitability of the left motor cortex by recording EMG over the muscles involved in the right-hand effector. In this way, then, it was possible to measure whether emotional stimuli would selectively influence response-related MEPs. Moreover, by administering TMS pulses at various intervals before the response, it was possible to examine the dynamics of the corticospinal excitability and to track the time course of the effect of emotion (Burle, Bonnet, Vidal, Possamaï, \& Hasbroucq, 2002; van den Wildenberg et al., 2010). After completing the TMS session, each subject rated the emotional stimuli on a valence scale.

We investigated the extent to which emotion-induced modulation of action readiness would show a dynamic buildup toward the point in time at which the imminent action was to be executed. We hypothesized that the amplitudes of the MEP would be larger when the subjects viewed emotional stimuli than when they viewed neutral stimuli, as Hajcak et al. (2007) found. Moreover, we hypothesized that this effect would be obtained independently of the hand used to make a response. That is, emotional stimuli would be associated with a general increase in corticospinal excitability. In accordance with Hajcak et al.'s findings, we did not expect differential MEP amplitudes between unpleasant and pleasant pictures. Furthermore, we hypothesized that the MEP amplitude would increase as the timing of the TMS pulse approached the time of the overt response. Replicating typical findings, we hypothesized that this effect would be obtained for the hand 
involved in the response only (e.g., Burle et al., 2002). Importantly, we predicted an interaction between the effects of emotional stimuli and TMS interval, such that the impact of emotional stimuli on the motor system would increase as TMS pulses were presented closer in time to the overt response. A main effect of emotional stimuli, irrespective of TMS timing, would confirm the tonic emotional modulation effect reported by Hajcak et al. Conversely, a dynamic modulation would be expressed by a progressively increasing effect of emotional stimuli as a function of time, but only for the hand involved in the movement, not for the noninvolved hand.

In summary, we predicted that emotional task-irrelevant stimuli would increase MEP amplitudes in both the hand involved in the response and the noninvolved hand and that MEP amplitude in the response hand would increase over time. Finally, we expected that this increase would be larger during the presentation of emotional pictures than during presentation of neutral pictures.

\section{METHOD}

\section{Subjects}

Eleven healthy volunteers ( 4 males; $M=28.7$ years of age, $S D=$ 5.8 years) with no relevant psychiatric or neurological history participated in the experiment. All had normal or corrected-to-normal vision. Written informed consent was obtained from each individual prior to participation. Four subjects were excluded from analysis due to technical/recording difficulties or malfunctions; thus, the final sample consisted of 7 individuals. This study was approved by the local Ethics Committee and was conducted in accordance with relevant laws and institutional guidelines.

\section{Stimuli}

In total, 120 pictures were selected out of the IAPS (Lang, Bradley, \& Cuthbert, 1999), of which 40 were unpleasant scenes (e.g., threat and mutilation), 40 were pleasant scenes (e.g., erotic scenes, sporting events), and 40 were neutral scenes (e.g., household objects, trees; see the Appendix). The pictures were identical to those used by Hajcak et al. (2007).

\section{Procedure}

The subjects were tested in a darkened room and were seated in a comfortable chair that supported hands and arms. The subjects gripped a plastic cylinder ( $3 \mathrm{~cm}$ in diameter, $11 \mathrm{~cm}$ in height $)$ in each hand; these were fixed on a table to the left and right of the body midline. The distal phalanx of the thumbs rested on a force sensor that was fixed on the top of the cylinder. To initiate a trial, the pressure on the force sensor needed to be in the range of an isometric precontraction force, with an upper and lower bound $(10-20 \mathrm{~N})$. This self-initiation procedure generated background EMG activity. A fixation cross appeared on the screen when the precontraction force entered the force window, crossing the lower bound of $10 \mathrm{~N}$. If the precontraction force was too weak and dropped below this value within $1 \mathrm{sec}$, the fixation cross extinguished, and the trial was paused until the subjects reentered the force window. If the subjects pressed too hard and the precontraction force crossed the upper bound of the force window fixed at $20 \mathrm{~N}$, the experiment was paused, and the subjects had to release the force button and reenter the force window. When the precontraction force had been maintained between 10 and $20 \mathrm{~N}$ for $1 \mathrm{sec}$, the trial started. The trials were terminated by a firm press with one of the two thumbs on the force sensor. Responses were practiced during a practice session. Response force was recorded and digitized online (A/D rate, $2 \mathrm{kHz}$ ). RT was defined as the moment in time at which the response force first exceeded $40 \mathrm{~N}$.

\section{RT Task}

The subjects performed a choice RT task. The task was first practiced on a block of 120 trials to familiarize the subject with the task and the self-initializing procedure controlled by the force buttons. The practice block was followed by two calibration blocks of 120 trials each to obtain individual RT distributions. These calibration data were used to set the timing of TMS pulses for each individual in the TMS session.

Each trial started with the presentation of an IAPS picture in the center of the screen. After $1 \mathrm{sec}$, two geometrical figures appeared on each side of the picture, and the subjects had to decide whether these figures were identical or different. The figures could be either a square or a circle (see Figure 1). If the figures were similar (i.e., congruent trial), either both squares or both circles, the subjects had to press with one hand - for example, the right. They were instructed to press with their other hand (in this case, the left) if the figures were different (i.e., incongruent trial) - for example, a square paired with a circle. The mapping of congruency to response hand was counterbalanced across subjects. Upon a response, the fixation cross was presented again for $1 \mathrm{sec}$, after which the trial ended and the screen went blank again. After each trial, the subjects were asked to briefly release the force buttons and to reenter the force window to start the next trial. Task instructions stressed the importance of fast and accurate responding.

The pictures were presented in color and were given in a blocked design. The stimuli were presented in two consecutive blocks of trials of similar emotional valence (e.g., two blocks of pleasant pictures, two blocks of unpleasant pictures, and two blocks of neutral pictures). The order of these pairs of blocks was counterbalanced between subjects.

The experiment consisted of a practice session in which the peripheral imperative figures were shown without central IAPS pictures, followed by a calibration session, a TMS session, and a picture-rating session. The TMS session contained 12 blocks of 120 trials and was completed in about $3.5 \mathrm{~h}$. In total, the subjects were presented with 1,440 trials $-6(5$ TMS intervals + no TMS pulse $) \times$ 2 (congruent and incongruent trials) $\times 3$ (pleasant, unpleasant, and neutral) - amounting to a total of 40 replications per cell.

\section{Picture Ratings}

The rating session of the IAPS pictures took place on a separate day after the TMS session and was completed in about $15 \mathrm{~min}$. The 120 pictures were presented randomly on a computer screen for $4 \mathrm{sec}$. After the presentation of a picture, a valence scale was shown, where 1 indicated very pleasant and 9 indicated very unpleasant. The cursor started randomly at one of the numbers on the scale, and the subjects had to move the cursor to their number of preference and press the Enter button to view the next picture.

Because valence is subjective, individual valence ratings of the pictures were used to categorize single trials. For each subject, the distribution of valence ratings was used to determine individual emotional categories. The median rating value was used to classify the neutral category, and the left and right tails of the distribution of valence scores were used to categorize the pleasant and unpleasant pictures, respectively. To obtain a balanced distribution, each emotional category contained about $25 \%$ of the trials; the remaining trials were not analyzed further. For example, if the median for neutral pictures was 5 , only trials that contained a picture that was rated with 5 were included in the analyses. For the pleasant pictures, only those that were rated with extreme valence scores-for example, 3 or less - were included. This procedure ensured comparable trial numbers between individuals and between emotion categories.

\section{TMS Procedure}

Magnetic stimulation was delivered using a figure-eight-shaped coil with a 7-cm diameter connected to a MagStim Rapid ${ }^{2}$ (Magstim, Whitland, U.K.) with 2.2-T maximum output. The coil was held in a swan neck that was bolted to the back of the chair. A head frame maintained a light and comfortable restraint of the subject's head. The coil was placed tangentially on the head over the left M1, 

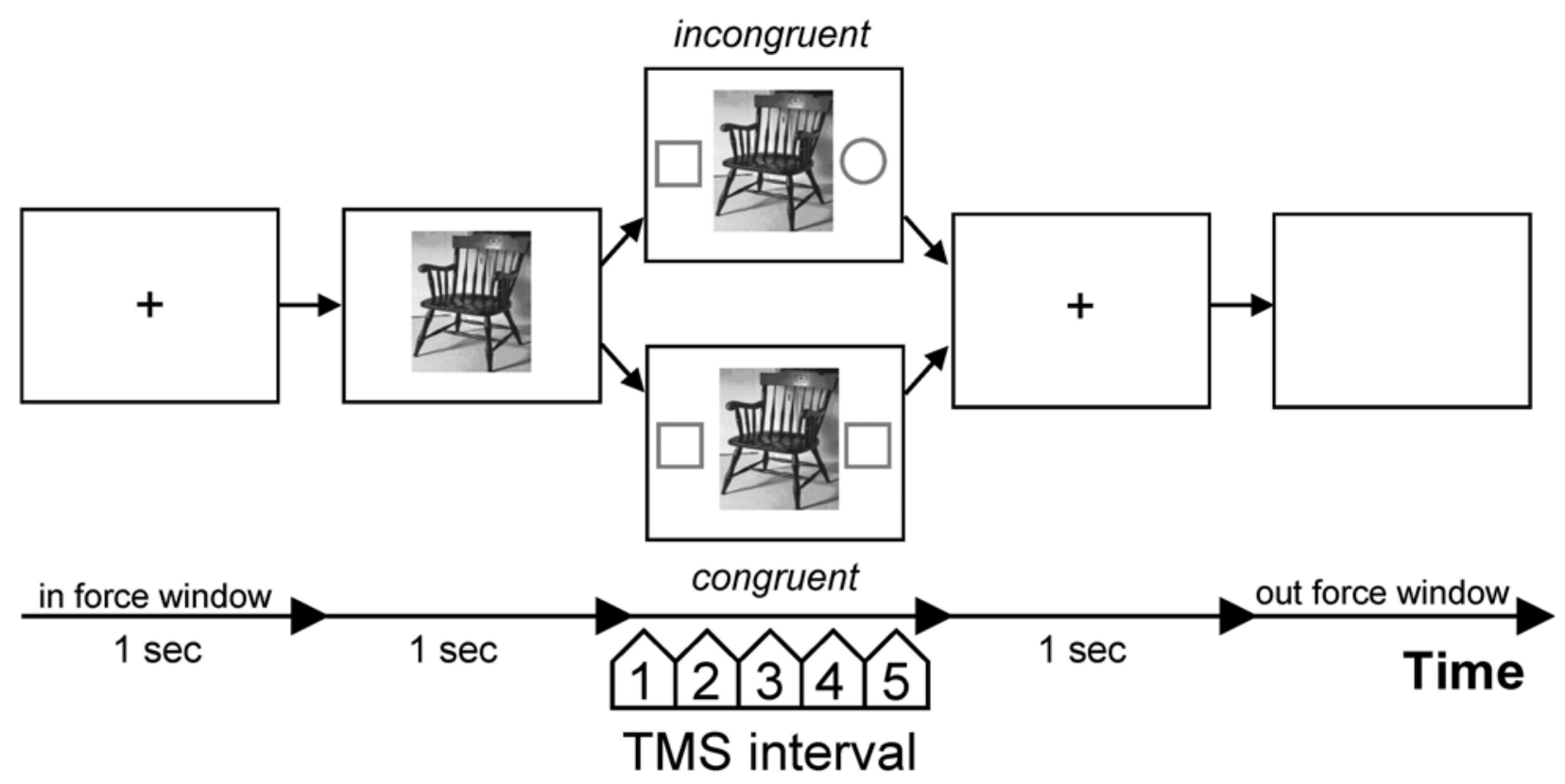

Figure 1. Procedure of a trial. To start the trial, the subjects had to press both response buttons within the force window. Then a fixation cross appeared for $1 \mathrm{sec}$, followed by a task-irrelevant picture. After $1 \mathrm{sec}$, two peripheral figures appeared that required either a left- or a right-hand response, on the basis of the congruency of the peripheral figures. After the response, the fixation cross appeared again for 1 sec, followed by a blank screen. TMS, transcranial magnetic stimulation.

with the induced current passing forward over the motor representation of the right hand. Next, the coil was moved to determine the location at which a visible TMS-induced flexion of the right thumb could be obtained. After the correct location had been found, the stimulation intensity necessary for subthreshold EMG activity was determined individually in a series of adjustment trials. The subjects exerted a constant press on the force sensor ranging from 10 to $20 \mathrm{~N}$ during 2 min while receiving 10 TMS pulses that were delivered in a pseudorandom temporal sequence. EMG signals recorded over the muscles of the right thumb were time-locked to TMS onset and were inspected visually. On the basis of the adjustment trials, the intensity of TMS was determined individually on the basis of the quality of the MEP, the absence of artifacts, and the absence of overt TMS-induced thumb movements (see Burle et al., 2002). More than half of the 10 TMS pulses should elicit an MEP of at least $50 \mathrm{mV}$. After the adjustment trials, individual stimulation intensity was kept constant throughout the experiment, within a range of $52 \%-65 \%$ of maximum output intensity for all the subjects tested $(M=59 \%)$.

TMS timing. TMS pulses were delivered between the presentation of the imperative geometrical figures and the time of responding. The RT results obtained in the two behavioral calibration blocks were used to determine the TMS intervals used in the TMS session. TMS was delivered on 1,200 trials ( $86 \%$ ); no pulse was presented on the remaining 240 trials. The timing of the TMS pulses was calculated individually at one of the five equidistant time points ( 240 pulses at each time point). This divided the interval between the onset of the peripheral figures and the value determined by the mean of the first decile of the RT distribution recorded in the calibration session. The minimum TMS interval was $47 \mathrm{msec}$, and the maximum was $378 \mathrm{msec}$.

\section{EMG Recordings}

The subjects positioned the distal phalanx of each thumb on the corresponding force sensor. Both arms rested on the table as comfortably as possible. The response was an isometric press of the right or left thumb on the corresponding sensor; it was measured as a force signal and was digitized online at $2 \mathrm{kHz}$. The EMG activity of the flexor pollicis brevis of the right and left hands was recorded with two $\mathrm{Ag} / \mathrm{AgCl}$ electrodes on each hand, $6 \mathrm{~mm}$ in diameter, glued $20 \mathrm{~mm}$ apart on the skin of the thenar eminence. The EMG was band-pass filtered between $10 \mathrm{~Hz}$ and $1 \mathrm{kHz}$ (Neuroscan, Inc.) and amplified (gain 10,000).

EMG activity and force signals were stored on disk for offline analyses, during which each trial was displayed on a computer screen with the EMG signal aligned to the onset of the imperative stimuli. Each TMS trial was inspected visually. This visual inspection allowed us to discard trials on which TMS was delivered during voluntary EMG activity (3.1\% of all trials, on average, across individuals). For the remaining single trials, MEP amplitude was scored as the maximal peak-to-peak difference following the TMS pulse. MEPs were not clearly discernable from noise on roughly half of the trials. These trials were not excluded, in order to obtain a sufficient number of trials for a reliable signal-to-noise ratio. The MEPs evoked during the series of adjustment trials served to define the time intervals during which the peak-to-peak value of the MEP was computed on each stimulation trial, which was 20-40 msec after the TMS pulse. The average MEP peak latency in this study was $26 \mathrm{msec}(S D=0.5 \mathrm{msec})$.

\section{RESULTS}

\section{Behavioral Data}

Picture-rating scores. The subjects judged the picture set in high accordance with the original (IAPS) valence ratings (see Table 1). Note that a high value on the valence scale indicates unpleasant, and a low value indicates pleasant. A repeated measures ANOVA confirmed that the valence ratings differed significantly between emotion categories $[F(2,12)=$ $165.95, p<.0001]$. A Helmert contrast between the neutral and the emotional conditions and a simple contrasts analysis between the conditions indicated that unpleasant, neutral, and pleasant pictures were rated as reliably different from one another on the valence dimension $(p \mathrm{~s}<.001)$.

RT data. The behavioral results are summarized in Table 2. Incidental RTs longer than $1,000 \mathrm{msec}$ were removed from the analyses. Trial type (congruent or incongru- 
Table 1

Subjective and Normative Mean Valence Rating Scores on a Scale Ranging From 1 (Very Pleasant) to 9 (Very Unpleasant)

\begin{tabular}{|c|c|c|c|c|c|c|}
\hline & \multicolumn{6}{|c|}{ Valence Category } \\
\hline & \multicolumn{2}{|c|}{ Unpleasant } & \multicolumn{2}{|c|}{ Neutral } & \multicolumn{2}{|c|}{ Pleasant } \\
\hline & $M$ & $S E$ & $M$ & $S E$ & $M$ & $S E$ \\
\hline Subjective ratings & 8.16 & 0.21 & 5.00 & 0 & 2.57 & 0.23 \\
\hline Normative ratings* & 6.77 & 0.07 & 5.07 & 0.06 & 2.02 & 0.15 \\
\hline
\end{tabular}

${ }^{*}$ For the subjective categories.

Table 2

Means and Standard Errors for

Reaction Times (RTs, in Milliseconds) and

Proportional Error Rates per Congruency (Congruent

vs. Incongruent), Subjective Emotional Valence (Unpleasant, Neutral, Pleasant), and Trial Type (TMS Pulse vs. No TMS Pulse)

\begin{tabular}{lccccc}
\hline & \multicolumn{2}{c}{ RT } & & \multicolumn{2}{c}{ Errors } \\
\cline { 2 - 3 } \cline { 5 - 6 } & $M$ & $S E$ & & $M$ & $S E$ \\
\hline Congruent & 493 & 26 & & .09 & .01 \\
Incongruent & 517 & 27 & & .07 & .01 \\
Unpleasant & 503 & 28 & & .08 & .01 \\
Neutral & 507 & 26 & & .08 & .01 \\
Pleasant & 510 & 28 & & .08 & .01 \\
TMS pulse & 503 & 25 & .06 & .01 \\
No TMS pulse & 514 & 30 & .08 & .01 \\
\hline
\end{tabular}

ent) did not have an effect on RTs $[F(1,6)=2.87, p=.14]$ or error rates $(F<1)$. The effects of emotional valence on RTs and accuracy also were not significant $(F \mathbf{S}<1)$. A trend was found for the effect of the TMS pulses on error rates; slightly more errors were made when a TMS pulse was given than when no pulse was given $[F(1,6)=5.80, p=.05]$. This, however, had no effect on RT $[F(1,6)=2.21, p=.19]$.

\section{EMG Data}

Because of considerable intersubject variability, individual MEP amplitudes were standardized using $z$ scores based on individual means and standard deviations computed over all the stimulation trials (cf. Burle et al., 2002). If Mauchly's test of sphericity was significant and sphericity could not be assumed, the $p$ value after Huynh-Feldt correction of the degrees of freedom is reported (uncorrected $d f$ s are reported, however, for ease of reading).

A main effect of the emotional valence of the pictures on MEP amplitude was expected, independently of whether the hand was involved or noninvolved in the response. It was hypothesized that emotional pictures would elicit higher MEP amplitudes than would neutral pictures, as reported by Hajcak et al. (2007).

Therefore, a repeated measures ANOVA with emotional valence (pleasant, neutral, or unpleasant), TMS interval $(1,2,3,4$, or 5), and hand involvement (involved or noninvolved) as within-subjects factors was performed. To compare the mean MEP amplitudes between the neutral condition and the two emotional conditions, a Helmert contrast analysis was used. A significant difference between the emotional conditions and the neutral condition was obtained; emotional pictures elicited higher MEP amplitudes than did neutral scenes $[F(1,6)=8.80, p=$ $.03]$. There was no difference in the mean MEP amplitude between unpleasant and pleasant emotional conditions $(F<1)$. This is consistent with the pattern of findings reported by Hajcak et al. (2007) (see Figure 2).

Another factor that was manipulated was TMS interval - that is, the time at which a TMS pulse was given. Mean MEP amplitude increased over time $[F(4,24)=$ $9.84, p<.001]$. The third factor that could influence mean MEP amplitude was hand involvement. As was predicted, mean MEP amplitude was significantly higher when the hand performed the response than when the hand was not involved in responding $[F(1,6)=14.82, p=.01]$. The effect of interest was the three-way interaction between the emotional valence, TMS interval, and hand involvement factors. The visual impression of this interaction effect on MEP amplitude, displayed in Figure 3, was confirmed statistically $[F(8,48)=6.70, p<.001]$.

The main goal of this study was to investigate a possible dynamic modulation of motor excitability by emotional stimuli. We hypothesized that the MEP amplitude for the involved hand would increase progressively toward the execution of the response and that this increase would be larger for the pictures with an emotional valence than for neutral pictures. This would imply that hand involvement moderates the interaction effect between emotional valence and TMS interval. Therefore, we looked at the interaction between emotional valence and TMS interval separately for the hand's being involved and for the hand's not being involved in responding. Helmert contrast analyses were used to compare the emotional conditions and the neutral condition and to compare earlier TMS intervals with later TMS intervals.

No interaction effects were found for the hand that was not involved in the response (all $F_{\mathrm{s}}<1.44$, all $p \mathrm{~s}>$.28). By contrast, if the hand was involved in the response, the twoway interaction was present, starting from the second TMS interval and onward, when the neutral condition was compared with the two emotional conditions (all $F_{\mathrm{s}}>6.8$, all $p \mathrm{~s}<.04)$. An interaction effect between emotional valence and TMS interval was also found when the two emotional conditions were specifically contrasted with each other.

\section{Average MEP Amplitude}

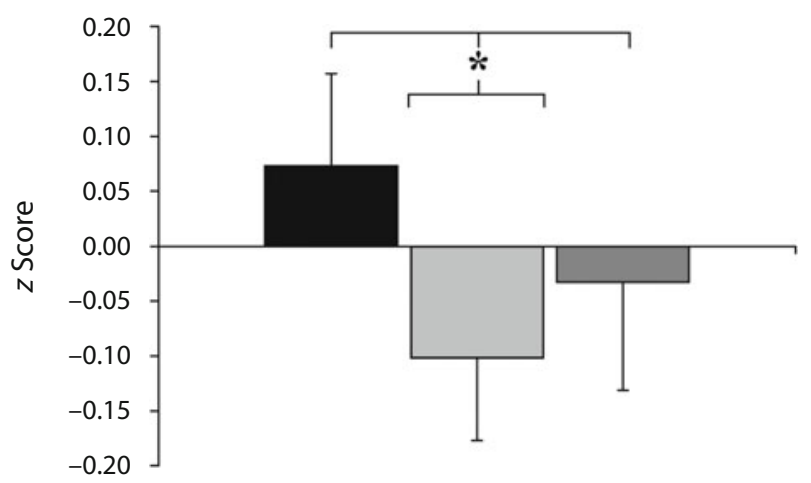

Unpleasant Neutral Pleasant

Figure 2. Overall mean motor-evoked potential (MEP) amplitudes in $z$ scores per valence condition (unpleasant, neutral, or pleasant). $\quad * p<.05$. 
MEP Amplitude per TMS Interval

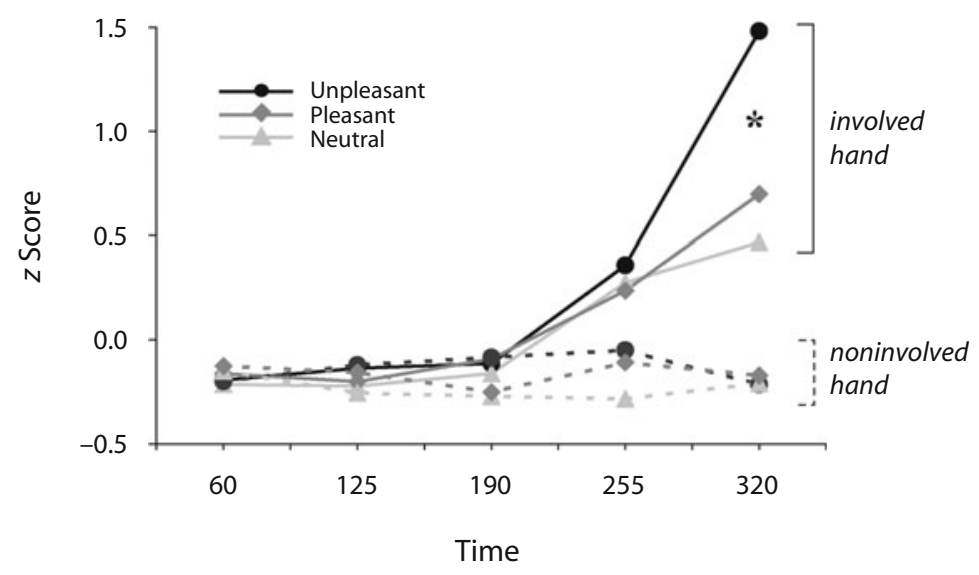

Figure 3. Mean motor-evoked potential (MEP) amplitudes in $z$ scores for each transcranial magnetic stimulation (TMS) interval $(60,125,190,255$, and $320 \mathrm{msec}$ ) per valence condition (unpleasant, neutral, or pleasant) and hand (involved or noninvolved in the response). ${ }^{*} p<.05$.

Unpleasant pictures elicited significantly higher MEPs than did pleasant pictures when the first TMS interval was compared with the later TMS intervals overall $[F(1,6)=$ $8.98, p=.024]$ and also when the fourth and fifth TMS intervals were compared $[F(1,6)=9.14, p=.023]$.

Apparently, hand involvement and TMS interval moderated the effect of emotional valence on MEP amplitude. Therefore, an extra analysis was performed that focused on the fifth TMS time point (see Figure 4). The main effect of emotional valence on the MEP amplitude at this longest TMS interval for the hand involved in responding was significant $[F(2,12)=9.31, p<.01]$. Both pleasant and unpleasant pictures elicited higher MEP amplitudes at this time point than did neutral pictures $[F(1,12)=9.45$, $p=.02]$. Additional simple contrast analyses showed that pleasant pictures did not differ from neutral pictures $[F(1,6)=1.00, p=.36]$, but unpleasant pictures differed significantly from neutral pictures $[F(1,6)=16.41, p<$ $.01]$, as well as from pleasant pictures $[F(1,6)=9.19, p<$ $.05]$, at this latest TMS interval.

It could be argued that the differential effects of emotional valence on MEP amplitude are caused by differences in tonic muscle tension between emotion conditions. TMSevoked MEPs are increased when muscles are contracted (Hess, Mills, \& Murray, 1986, 1987). To test this alternative hypothesis, we computed the mean pre-TMS EMG activity recorded in the window starting $150 \mathrm{msec}$ and ending $50 \mathrm{msec}$ before the TMS pulse. The EMG values were entered into a repeated measures ANOVA with Helmert's contrasts for both involved and noninvolved hands. This analysis yielded no significant effects for emotional valence on the pre-TMS EMG $(p>.36)$, suggesting that tonic muscle contraction was comparable across emotional conditions.

\section{DISCUSSION}

Our main result was that emotional task-irrelevant pictures elicited higher MEP amplitudes following TMS pulses than did neutral pictures. MEP amplitude was largest when the subjects viewed unpleasant pictures, followed by the presentation of pleasant pictures. The smallest MEP amplitudes were seen when the subjects were watching neutral stimuli. Thus, task-irrelevant emotional stimuli increased corticospinal excitability, evident through a modulation of the size of the MEP amplitude. This finding fits well with the results from previous studies (Avenanti et al., 2005; Avenanti et al., 2009; Baumgartner et al., 2007; Fecteau et al., 2008; Hajcak et al., 2007; Oathes et al., 2008; Oliveri et al., 2003; Schutter et al., 2008). Increased activity in the motor areas of the brain during emotional processing has also been reported in PET studies (Bremner et al., 1999; Rauch et al., 1996). Furthermore, modulation of MEP amplitudes has been reported previously in other contexts - for example, during imagined movement (Fadiga et al., 1999; Rossi, Pasqualetti, Tecchio, Pauri, \& Rossini, 1998) and following instructions to prepare for movement (Hoshiyama et al., 1997).

\section{MEP Amplitude at TMS Interval 5}

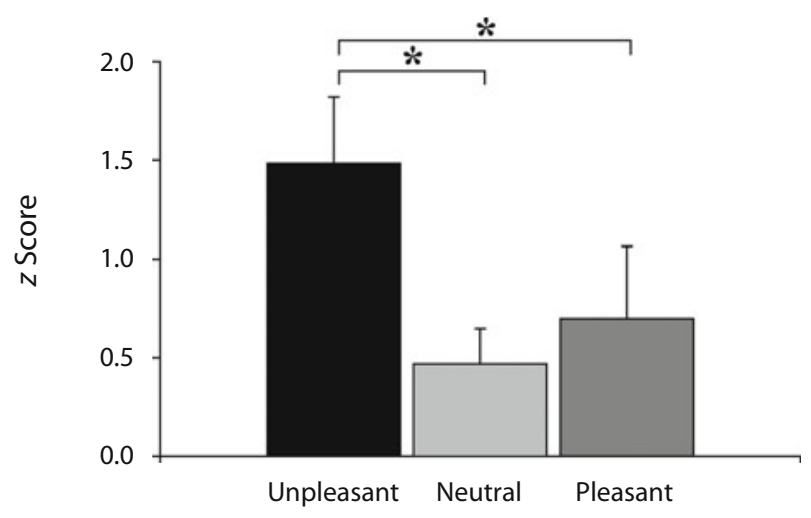

Figure 4. Mean motor-evoked potential (MEP) amplitudes in $z$ scores for the involved hand at the longest transcranial magnetic stimulation (TMS) interval (i.e., 320 msec). ${ }^{*} p<.05$. 
Our results also demonstrate that during action preparation, corticospinal excitability associated with the response hand gradually increased. This observation is in line with the findings of previous studies (Leocani et al., 2000; Nikolova et al., 2006; Rossini et al., 1988; van den Wildenberg et al., 2010). However, our main interest was in the possible effect of emotional processing on the dynamics of corticospinal excitability and in extending previous results that showed a tonic effect of emotion on the action system. Indeed, we found that the increase in corticospinal excitability over time was amplified by emotional task-irrelevant stimuli. This is the first report of such a result, since this is (to our knowledge) the first study that has shown the dynamic effect of emotional processing on corticospinal excitability.

We also observed a significant difference between the effects of unpleasant and pleasant stimuli on action readiness. The effect on corticospinal excitability was stronger for the unpleasant pictures than for the pleasant pictures. This difference has not been reported in previous studies (Baumgartner et al., 2007; Hajcak et al., 2007). This discrepancy might well be explained by the increased temporal sensitivity and specificity achieved in the present study; here, TMS was applied at various intervals preceding the response. Unpleasant pictures elicited higher MEP amplitudes than did pleasant pictures only at the longest TMS interval.

\section{Alternative Accounts}

An important concern is that the emotional categories of the stimuli may differ not only in their emotional valence, but also in terms of content. Neutral stimuli mostly depict static objects, whereas pleasant and unpleasant pictures mostly show humans involved in dynamic situations - that is, engaged in some mouth/facial or hand/arm activity or operating an object such as a gun or a knife. Observation of others' (emotionally neutral) actions increases the MEP amplitude (Fadiga, Fogassi, Pavesi, \& Rizzolatti, 1995; Montagna, Cerri, Borroni, \& Baldissera, 2005), as does observation of implied motion stimuli (Urgesi, Moro, Candidi, \& Aglioti, 2006). As a result, the present MEP patterns might also be explained by the difference in action content of the stimuli. However, the fact that the pleasant and unpleasant pictures used in our study were highly similar in depicting humans and human actions renders an explanation of the observed difference in MEP amplitude between unpleasant and pleasant conditions in terms of action content unlikely.

It should be noted that although MEPs were not always clearly distinguishable by eye, these trials were included in the analysis nonetheless, to increase the signal-to-noise ratio. In addition, it was not possible to analyze silent period durations, because the silent period was not clearly discernable from EMG background noise on most of the trials. Although the silent period was not included as a dependent measure in the present study, it would be informative to quantify the effects of emotional valence on the length of the silent period and to investigate the involvement of GABA-b mediated cortical inhibitory circuits (see also Di Lazzaro et al., 2005; Stefan, Wycislo, \& Classen, 2004; van den Wildenberg et al., 2010). Future studies might employ a higher TMS intensity than the one used in the present study to improve the discernability of the MEPs and the incidence of the silent period.

Another direction for further research is to disentangle where, in the corticospinal tract, the emotional modulation takes place. Does the increase of the MEP amplitude reflect an increase in primary motor cortex excitability, an enhanced spinal excitability, or both? Effects of emotional stimuli on the spinal reflex have been reported in previous studies (Bonnet, Bradley, Lang, \& Requin, 1995; Both, Everaerd, \& Laan, 2003). To address this question, appropriate techniques, such as paired-pulse TMS or methods demonstrating spinal and peripheral motor excitability (e.g., H-reflex, F- and M-waves), may be used.

In sum, the present findings suggest that corticospinal excitability is elevated by induced affect - in particular, by negative emotional valence - and that this effect becomes progressively more pronounced just prior to responding. These patterns support the notion that emotional stimuli, even when task irrelevant, not only trigger affective processing, but also modulate readiness for action in a dynamic way.

\section{AUTHOR NOTE}

This research was supported by grants from the Netherlands Organization for Scientific Research (NWO) to the second and last authors. We thank Dilene van Campen for her assistance in testing the subjects. Thomas Pronk, Bert Molenkamp, and Marcus Spaan are gratefully acknowledged for their technical support. Correspondence concerning this article should be addressed to A. M. van Loon, Department of Psychology, University of Amsterdam, Roetersstraat 15, 1018 WB Amsterdam, The Netherlands (e-mail: anouk.vanloon@gmail.com).

\section{REFERENCES}

Avenanti, A., Bueti, D., Galati, G., \& Aglioti, S. M. (2005). Transcranial magnetic stimulation highlights the sensorimotor side of empathy for pain. Nature Neuroscience, 8, 955-960. doi:10.1038/nn1481

Avenanti, A., Minio-Paluello, I., Bufalari, I., \& Aglioti, S. M. (2009). The pain of a model in the personality of an onlooker: Influence of state-reactivity and personality traits on embodied empathy for pain. NeuroImage, 44, 275-283. doi:10.1016/j.neuroimage.2008.08.001

Baumgartner, T., Willi, M., \& JÄNCKe, L. (2007). Modulation of corticospinal activity by strong emotions evoked by pictures and classical music: A transcranial magnetic stimulation study. NeuroReport, 18, 261-265. doi:10.1097/WNR.0b013e328012272e

BoHNING, D. E. (2000). Introduction and overview of TMS physics. In M. S. George \& R. H. Belmaker (Eds.), Transcranial magnetic stimulation in neuropsychiatry (pp. 13-44). Washington, DC: American Psychiatric Press.

Bonnet, M., Bradley, M. M., Lang, P. J., \& Requin, J. (1995). Modulation of spinal reflexes: Arousal, pleasure, action. Psychophysiology, 32, 367-372. doi:10.1111/j.1469-8986.1995.tb01219.x

Both, S., Everaerd, W., \& LAan, E. (2003). Modulation of spinal reflexes by aversive and sexually appetitive stimuli. Psychophysiology, 40, 174-183. doi:10.1111/1469-8986.00019

Bremner, J. D., Staib, L. H., Kaloupek, D., Southwick, S. M., Soufer, R., \& Charney, D. S. (1999). Neural correlates of exposure to traumatic pictures and sound in Vietnam combat veterans with and without posttraumatic stress disorder: A positron emission tomography study. Biological Psychiatry, 45, 806-816. doi:10.1016/S0006 $-3223(98) 00297-2$

Burle, B., Bonnet, M., Vidal, F., Possamaï, C., \& Hasbroucq, T. (2002). A transcranial magnetic stimulation study of information processing in the motor cortex: Relationship between the silent period and the reaction time delay. Psychophysiology, 39, 207-217. doi:10.1111/1469-8986.3920207 
Coombes, S. A., Gamble, K. M., Cauraugh, J. H., \& Janelle, C. M. (2008). Emotional states alter force control during a feedback occluded motor task. Emotion, 8, 104-113.

DARWIN, C. (1965). The expression of the emotions in man and animals. Chicago: University of Chicago Press. (Original work published 1872)

Di Lazzaro, V., Oliviero, A., Saturno, E., Dileone, M., Pilato, F., NARDONE, R., ET AL. (2005). Effects of lorazepam on short latency afferent inhibition and short latency intracortical inhibition in humans. Journal of Physiology, 564, 661-668. doi:10.1113/jphysiol .2004 .061747

Duckworth, K. L., Bargh, J. A., Garcia, M., \& Chaiken, S. (2002). The automatic evaluation of novel stimuli. Psychological Science, 13, 513-519. doi:10.1111/1467-9280.00490

Erthal, F. S., De Oliveira, L., Mocaiber, I., Pereira, M. G., MachadoPinheiro, W., Volchan, E., \& Pessoa, L. (2005). Load-dependent modulation of affective picture processing. Cognitive, Affective, \& Behavioral Neuroscience, 5, 388-395. doi:10.3758/CABN.5.4.388

Fadiga, L., Buccino, G., Craighero, L., Fogassi, L., Gallese, V., \& PAVESI, G. (1999). Corticospinal excitability is specifically modulated by motor imagery: A magnetic stimulation study. Neuropsychologia, 37, 147-158. doi:10.1016/S0028-3932(98)00089-X

Fadiga, L., Fogassi, L., Pavesi, G., \& Rizzolatti, G. (1995). Motor facilitation during action observation: A magnetic stimulation study. Journal of Neurophysiology, 73, 2608-2611.

Fecteau, S., Pascual-Leone, A., \& Théoret, H. (2008). Psychopathy and the mirror neuron system: Preliminary findings from a nonpsychiatric sample. Psychiatry Research, 160, 137-144. doi:10.1016/ j.psychres.2007.08.022

FrIJdA, N. H. (1986). The emotions. Cambridge: Cambridge University Press.

FrIJDA, N. H. (2007). The laws of emotion. Mahwah, NJ: Erlbaum.

Hajcak, G., Molnar, C., George, M. S., Bolger, K., Koola, J., \& NAHAS, Z. (2007). Emotion facilitates action: A transcranial magnetic stimulation study of motor cortex excitability during picture viewing. Psychophysiology, 44, 91-97. doi:10.1111/j.1469-8986 .2006.00487.x

Hess, C. W., Mills, K. R., \& Murray, N. M. (1986). Magnetic stimulation of the human brain: Facilitation of motor responses by voluntary contraction of ipsilateral and contralateral muscles with additional observations on an amputee. Neuroscience Letters, 71, 235-240.

Hess, C. W., Mills, K. R., \& Murray, N. M. (1987). Responses in small hand muscles from magnetic stimulation of the human brain. Journal of Physiology, 388, 397-419.

Hoshiyama, M., Kakigi, R., Koyama, S., Takeshima, Y., WaTANabe, S., \& Shimojo, M. (1997). Temporal changes of pyramidal tract activities after decision of movement: A study using transcranial magnetic stimulation of the motor cortex in humans. Electroencephalography \& Clinical Neurophysiology, 105, 255-261. doi:10.1016/ S0924-980X(97)00019-2

Lang, P. J., Bradley, M. M., \& Cuthbert, B. N. (1999). International Affective Picture System: Instruction manual and affective ratings (Tech. Rep. A-4). Gainesville: University of Florida, Center for Research in Psychophysiology.
Leocani, L., Cohen, L. G., Wassermann, E. M., Ikoma, K., \& HalLETT, M. (2000). Human corticospinal excitability evaluated with transcranial magnetic stimulation during different reaction time paradigms. Brain, 123, 1161-1173.

LEWIS, M. D. (2005). Bridging emotion theory and neurobiology through dynamic systems modeling. Behavioral \& Brain Sciences, 28, 169-245. doi:10.1017/S0140525X0500004X

Montagna, M., Cerri, G., Borroni, P., \& Baldissera, F. (2005). Excitability changes in human corticospinal projections to muscles moving hand and fingers while viewing a reaching and grasping action. European Journal of Neuroscience, 22, 1513-1520. doi:10.1111/j.1460 -9568.2005.04336.x

Nikolova, M., Pondev, N., Christova, L., Wolf, W., \& Kossev, A. R. (2006). Motor cortex excitability changes preceding voluntary muscle activity in simple reaction time task. European Journal of Applied Physiology, 98, 212-219. doi:10.1007/s00421-006-0265-y

OAthes, D. J., BRuce, J. M., \& NitschKe, J. B. (2008). Worry facilitates corticospinal motor response to transcranial magnetic stimulation. Depression \& Anxiety, 25, 969-976. doi:10.1002/da.20445

Oliveri, M., Babiloni, C., FilipPi, M. M., Caltagirone, C., BabiLONI, F., CICINELli, P., ET AL. (2003). Influence of the supplementary motor area on primary motor cortex excitability during movements triggered by neutral or emotionally unpleasant visual cues. Experimental Brain Research, 149, 214-221. doi:10.1007/s00221-002-1346-8

Rauch, S. L., van der Kolk, B. A., Fisler, R. E., Alpert, N. M., OrR, S. P., Savage, C. R., ET AL. (1996). A symptom provocation study of posttraumatic stress disorder using positron emission tomography and script-driven imagery. Archives of General Psychiatry, 53, 380-387.

Rossi, S., Pasqualetti, P., Tecchio, F., Pauri, F., \& Rossini, P. M. (1998). Corticospinal excitability modulation during mental simulation of wrist movements in human subjects. Neuroscience Letters, 243, 147-151. doi:10.1016/S0304-3940(98)00088-3

Rossini, P. M., Zarola, F., Stalberg, E., \& Caramia, M. (1988). Premovement facilitation of motor-evoked potentials in man during transcranial stimulation of the central motor pathways. Brain Research, 458, 20-30. doi:10.1016/0006-8993(88)90491-X

Schutter, D. J. L. G., Hofman, D., \& Van Honk, J. (2008). Fearful faces selectively increase corticospinal motortract excitability: A transcranial magnetic stimulation study. Psychophysiology, 45, 345-348. doi:10.1111/j.1469-8986.2007.00635.x

Stefan, K., WyCislo, M., \& Classen, J. (2004). Modulation of associative human motor cortical plasticity by attention. Journal of Neurophysiology, 92, 66-72. doi:10.1152/jn.00383.2003

TERAO, Y., \& UgawA, Y. (2002). Basic mechanisms of TMS. Journal of Clinical Neurophysiology, 19, 322-343.

Urgesi, C., Moro, V., Candidi, M., \& Aglioti, S. M. (2006). Mapping implied body actions in the human motor system. Journal of Neuroscience, 26, 7942-7949. doi:10.1523/JNEUROSCI.1289-06.2006

van den Wildenberg, W. P. M., Burle, B., Vidal, F., Van der Molen, M. W., RidderinkHof, K. R., \& HasbroucQ, T. (2010). Mechanisms and dynamics of cortical motor inhibition in the stop-signal paradigm: A TMS study. Journal of Cognitive Neuroscience, 22, 225-239.

\section{APPENDIX}

The numbers of the IAPS pictures that were used were the following: pleasant, 1601, 2000, 2070, 2080, 2091, 2092, 2165, 2311, 2340, 4002, 4180, 4220, 4290, 4532, 4572, 4608, 4658, 4659, 4660, 4664, 4800, 4810, $5470,5621,5626,5628,7325,8021,8032,8080,8200,8210,8280,8320,8330,8370,8400,8465,8490,8540$; neutral, 2190, 2480, 2570, 2840, 2880, 5390, 5500, 5510, 5532, 5534, 5731, 5740, 5800, 5900, 7000, 7002, 7004, 7006, 7009, 7010, 7025, 7030, 7034, 7035, 7040, 7060, 7080, 7090, 7100, 7140, 7150, 7175, 7190, 7205, 7217, 7224, 7233, 7235, 7491, 7950; and unpleasant, 2800, 2900, 3051, 3102, 3110, 3261, 3530, 3550, 6230, $6242,6250,6260,6313,6350,6370,6510,6540,6560,6570,6571,6821,9040,9050,9253,9300,9400,9405$, $9410,9421,9433,9490,9520,9530,9570,9800,9810,9910,9911,9920,9921$. 